RESEARCH ETHICS

\title{
Eliminating the daily life risks standard from the definition of minimal risk
}

\section{B Resnik}

J Med Ethics 2005;31:35-38. doi: 10.1136/jme.2004.010470

The phrase "minimal risk," as defined in the United States' federal research regulations, is ambiguous and poorly defined. This article argues that most of the ambiguity that one finds in the phrase stems from the "daily life risks" standard in the definition of minimal risk. In this article, the author argues that the daily life risks standard should be dropped and that "minimal risk" should be defined as simply "the probability and magnitude of the harm or discomfort anticipated in research are not greater than those encountered during the performance of routine physical or psychological examinations or tests".

Correspondence to: Dr D B Resnik, National Institute of Environmental Health Science, National Institute of Health, Box 12233, NH06, Research Triangle Park, NC 27709; resnikd@niehs.nih.gov

Received 1 September 2004 Accepted for publication 6 September 2004
$\mathrm{T}$ he federal human research regulations define "minimal risk" as "the probability and magnitude of harm or discomfort anticipated in the research are not greater in and of themselves than those ordinarily encountered in daily life" (henceforth, "the daily life risks standard") or "during the performance of routine physical or psychological examinations or tests" (henceforth, "the routine tests standard"). ${ }^{1}$ Six different parts of the regulations use the concept of minimal risk, including parts that apply to (1) research on children; (2) research on prisoners; (3) research on fetuses; (4) procedures for altering the elements of informed consent; (5) procedures for waiving documentation requirements; and (6) expedited review of research. Although minimal risk plays a key role in the United States' (US) federal research regulations, it has generated considerable controversy. Several writers have argued that the concept is ambiguous and poorly defined. ${ }^{23}$ This article argues that most of the ambiguity that one finds in the phrase "minimal risk" stems from the daily life risks standard in the definition of minimal risk. This article argues that the daily life risks standard should be dropped and that "minimal risk" should be defined as simply "the probability and magnitude of the harm or discomfort anticipated in research are not greater than those encountered during the performance of routine physical or psychological examinations or tests".

\section{DEFINING RISK}

Risk can be viewed as the product of two components: (1) the probability of the harm; (2) the magnitude (or seriousness) of the harm. ${ }^{4}$ There are many different kinds of risk corresponding to the types of harm that can occur to a person. Among these include physiological risks, such as injury, disability, or death; psychological risks, such as depression or stress; social risks, such as stigmatisation; economic risk, such as loss of profits or property; and legal risks, such as civil or criminal liability. ${ }^{5}$ An aggregate risk is the sum of different, individual risks. For example, the risk of a research study that includes 10 venipunctures (blood draws) would have the risks associated with having 10 venipunctures, not the risks associated with have only one venipuncture. A study may be considered to be minimal risk only if the aggregate risks associated with the study are minimal.

In risk assessment, one attempts to assign values to the two components of risk. Very often it is possible to quantify risks by assigning numerical values to probabilities and harms. Sometimes it is not possible to obtain accurate and reliable quantitative (or numerical) measurements of risk, due to the inability to estimate probabilities, the inability to assign numerical values to harms, or both. When quantitative assessments of risk cannot be obtained, one may use qualitative (or categorical) assessments of risk. A qualitative assessment classifies risks according to categories, without measuring risk numerically. As it is usually difficult to obtain quantitative measurements of risk in human research, minimal risk should be viewed as a categorical assessment of risk. ${ }^{6}$

\section{MINIMAL RISK}

The concept of minimal risk plays an important role in protecting human subjects from research risks and serves as a kind of moral threshold in the federal regulations: if one crosses the threshold, then additional protections take effect. ${ }^{2}$ Most of the debate about minimal risk has focused on its role in paediatric research, where it protects children from exposure to research risks. ${ }^{78}$ The US regulations include special provisions for protecting children from research risks. The regulations distinguish between four categories of research on children:

- Research not involving greater than minimal risk. ${ }^{9}$

- Research involving greater than minimal risk but presenting the prospect of direct benefit to individual subject. ${ }^{10}$

- Research involving greater than minimal risk and no direct prospect of direct benefit to individual subjects, but likely to yield generalisable knowledge about the subject's disorder or condition. ${ }^{11}$

Abbreviations: IRB, institutional review board. 
- Research not otherwise approvable which presents an opportunity to understand, prevent, or alleviate a serious medical problem affecting the health or welfare of children. ${ }^{12}$

Minimal risk plays an important role in three of these four categories of research. Firstly, the concept serves as a threshold for allowing children to participate in research that does not directly benefit them or is not likely to yield knowledge about their disorder or condition. Children can participate in such research only if "no greater than minimal risk to children is presented" and "adequate provisions are made for soliciting the assent of the children and the permission of their parents or guardians." ${ }^{13}$ Parents cannot enrol their children in non-beneficial studies if the risks are beyond the threshold of minimal risk. This is a highly protective standard for decisions involving children, in which the government acts in loco parentis. ${ }^{2}$ Parents are not usually required, by law or ethics, to prevent their children from participating in activities that are more risky than daily activities or routine medical exams. Secondly, minimal risk helps to define the other risk categories in paediatric research, "greater than minimal risk" and "minor increase over minimal risk". Children may participate in greater than minimal risk research with no direct prospect of direct benefit to individual subjects, but likely to yield generalisable knowledge about the subject's disorder or condition, only if the research represents a minor increase over minimal risk.

Minimal risk also serves to protect prisoners and fetuses from research risks. The US regulations allow research on prisoners when the research involves a study of the "possible causes, effects, and processes of incarceration, and of criminal behavior" or a study of "prisons as institutional structures or of prisoners as incarcerated persons" and the research "presents no more than minimal risk and no more than inconvenience to the subjects". ${ }^{14}$ The US regulations require that pregnant women be excluded from research, unless the research is designed to meet the health needs of the mother and places the fetus only at the degree of risk necessary to meet those needs, or the risk to the fetus is minimal. ${ }^{15}$ The regulations provide far more protection for fetuses than the law normally requires. A pregnant woman can place her fetus at considerable risk, and even have an abortion, without running afoul of the law.

Minimal risk also plays a role in protecting all research subjects from risks in the provisions for waiving the informed consent requirements or the requirements for documenting consent. The US regulations require informed consent from the subject, or the subject's authorised representative. However, the regulations also contain a provision for waiving or altering the consent requirements. The regulations allow for a waiver or alteration of the informed consent requirements if:

- the research involves no more than minimal risk to the subjects;

- the waiver or alteration will not adversely affect the rights and welfare of the subjects;

- the research could not practicably be carried out without the waiver or alteration; and

- whenever appropriate, the subjects will be provided with additional pertinent information after participation. ${ }^{16}$

In this part of the regulations, the concept of minimal risk serves as a threshold for requiring informed consent. The regulations are stating, in effect, that when research risks are more than minimal, one needs the added protection afforded by the consent of the research subject or the subject's representative.
The US regulations also require that informed consent be documented using a written consent form. The regulations allow the institutional review board (IRB) to waive the documentation requirement in two situations, including a situation where "the research presents no more than minimal risk of harm to subjects and involves no procedures for which written consent is normally required outside of the research context" ${ }^{\prime 17}$ Here minimal risk serves as a threshold for requiring the added protection afforded by the documentation of informed consent.

Minimal risk protects research subjects from risk in the process of expedited review. The US regulations require IRBs to review research proposals and determine whether the proposals adhere to the federal regulations. IRBs also have the authority to monitor, oversee, alter, and stop research studies, if necessary. The regulations permit two types of review by IRBs: full IRB review and expedited review. Full IRB review requires a careful assessment of the proposal by the entire IRB and a vote. In expedited review, the IRB may designate the chair (or another reviewer) to make decisions on its behalf. The US regulations permit expedited review of research if either or both of the following apply: "some or all of the research appearing on the list and found by the reviewer(s) to involve no more than minimal risk" or "minor changes in previously approved research during the period (of one year or less) for which approval is authorized". ${ }^{18}$ The regulations are saying, in effect, that there is no need to provide the additional protection for human subjects afforded by full IRB review, if the research is deemed to be minimal risk.

\section{CRITICISMS OF THE CONCEPT OF MINIMAL RISK}

Research over the past two decades has revealed some significant inconsistencies with the interpretation of minimal risk. Empirical studies have shown that researchers do not agree on which risks should be classified as minimal. Two decades ago, a survey by Janofsky and Starfield found that paediatric researchers disagreed about whether venipuncture, arterial puncture, gastric and intestinal intubation, and tympanocentesis (piercing the ear drum) should be classified as minimal risk. ${ }^{19}$ A recent study by Shah and colleagues found that IRB chairpersons have different interpretations of the concept of minimal risk. ${ }^{20}$ Forty eight per cent of respondents in the study categorised magnetic resonance imaging with no sedation as minimal risk, while 35\% rated it as a minor increase over minimal risk, and 9\% viewed it as more than a minor increase over minimal risk. Forty four per cent classified a confidential survey of sexual activity as minimal risk, while $29 \%$ viewed it as a minor increase over minimal risk, and $19 \%$ considered it to be more than a minor increase over minimal risk. Twenty three per cent rated allergy skin testing as minimal risk, while $43 \%$ classified it as a minor increase over minimal risk, and $27 \%$ regarded it as more than a minor increase over minimal risk.

The variation that one finds in the interpretation of minimal risk results from the ambiguity in its definition in the federal research regulations. If the concept were more clearly defined, then there would be more agreement concerning its interpretation. One reason why the concept is ambiguous is there is no commonsense analogue of the concept of minimal risk. Minimal risk is an artifact of the federal research regulations, not a term drawn from commonsense morality or common law. Terms like "risk", "danger", "hazard", "harm", and "reasonable" have a place in our common, moral language, but the term "minimal risk" is a technical term that derives its meaning from the regulation of human research, not from ordinary language.

As minimal risk does not have a common meaning, it is important for the regulations to define the term clearly, but 
the regulations fail to do this. Kopelman's critiques of minimal risk help us to understand how the federal regulations fail to define the concept clearly. The definition of minimal risk found in the US research regulations consists of two different standards for classifying research risks: (1) the daily life risks standard and (2) the routine test standard. According to Kopelman, the daily life risks standard is ambiguous because it could be interpreted as referring to the daily life risks of the research subjects (a relativistic interpretation) or the daily life risks of a normal, healthy person (an absolute interpretation). ${ }^{3}$

Kopelman argues that there are serious problems with the relativistic interpretation, because different populations encounter different risks in daily life. ${ }^{321}$ A population of children from the inner city of Washington, DC faces more risks than a population from Ames, Iowa. A relativistic interpretation could allow researchers to exploit a population that faces very high risks in order to conduct research on that population. ${ }^{22}$ For example, a researcher might be able to argue that the Willowbrook hepatitis experiments, in which mentally disabled children were infected with hepatitis, posed only a minimal risk to them because hepatitis was endemic at the Willowbrook Institute. ${ }^{821}$

Kopelman also claims that using the relativistic interpretation could lead to inconsistencies that would be unjust to children, because IRBs might classify risks differently, depending on where the research is conducted. ${ }^{3}$ Sick children or children living in a dangerous environment might be exposed to more risks in research than healthy children or children living in safe environments. This would lead to an inequitable distribution of research risks: children who already face greater risks, as a result of their medical, social, or economic conditions, would bear a disproportionately high share of research risks. Many different authorities, including the National Commission, have held that the benefits and burdens of research should be distributed equitably. ${ }^{43}$ Furthermore, inconsistencies in the interpretation of minimal risk could also lead to exploitation of children (and IRBs) by encouraging researchers to "shop" for IRBs willing to regard protocols as minimal risk.

Freedman, Fuks, and Weijer reply to Kopelman's argument against the relativistic interpretation of the daily life risks standard by claiming the concept of minimal risk is a threshold for certain kinds of morally acceptable research, which should depend, in part, on community values. Where Kopelman sees the interpretation as leading to inconsistency and exploitation, they regard it as promoting flexibility and adaptability: Freedman, Fuks, and Weijer want to allow different communities (or cultures) to interpret daily life risks in light of their own values and standards. ${ }^{2}$ This is sort of like defining pornography in terms of community standards: what is considered pornography in Greenville, NC might not be considered pornography in New York City.

Although it is desirable for IRBs to take local conditions and standards into account when interpreting and applying the federal regulations, granting IRBs too much discretion threatens research subjects as well as the integrity and fairness of the research enterprise. Imagine what would happen if local communities interpreted the concept of "due process under the law" differently and the legal systems in different states accepted different interpretations of due process. This type of local variation could seriously threaten the political and moral rights of citizens and undermine fairness and integrity in the government. In the US, one standard of due process, defined by the US Constitution, applies to all localities. Likewise, one standard for minimal risk, defined by the US research regulations, should apply to different localities.
Although many people still defend the relativistic interpretation of daily life risks, a consensus in favour of the absolute standard is emerging. In a recent legal case dealing with the interpretation of minimal risk in paediatric research regulations, a Maryland appellate court endorsed the absolute interpretation of the daily life standard for minimal risk. ${ }^{24}$ The court also ruled that using children as test subjects to determine the effectiveness of lead abatement methods constituted more than minimal risk research, and that researchers had a legal duty to not enrol paediatric subjects in the research, because it provided the subjects no direct benefit nor did it benefit other subjects with their same disease or medical condition. The National Bioethics Advisory Commission has also endorsed the absolute interpretation for the daily life standard for minimal risk..$^{25}$ However, it remains to be seen whether this emerging consensus will have an affect on IRB deliberations.

Even the absolute interpretation of the daily life risks standard is not entirely unambiguous. ${ }^{3}$ How does one identify and describe the risks encountered by a normal, healthy person? The average person takes many different risks. He or she may travel by automobile, by airplane, by bicycle, or by boat. He or she may work in a factory, a convenience store, a hospital, or an office. He or she may walk along busy streets, engage in athletic activities (or no exercise at all), swim, eat or drink to excess, and maybe even use tobacco. Many of these risks taken by the typical person are much riskier than a venipuncture or many other medical tests. Weijer maintains that the risks encountered by the typical person are familiar to everyone, but I disagree. ${ }^{26}$ The risks encountered by the typical person are no more familiar to us than anything about the typical person-whether it is his or her age, sex, or race or ethnicity.

Health insurers know better than to sell individual insurance policies based on some understanding of the "average person". They attempt to gain information about that person's risk profile, such as age, health history, family history, lifestyle, so they can understand the risks encountered by that person. What makes sense for risk assessment in health insurance also makes sense for risk assessment in human research. To understand the risks associated with research, we should consider how risks associated the methods and procedures used in research affect individual research subjects, not typical or average subjects.

Although most of the debate about minimal risk has focused on the daily life risk standard, the routine test standard also has some ambiguity. What counts as a routine physical or psychological exam or test? As noted earlier, clinical researchers and IRB chairpersons do not agree whether venipunctures and other tests constitute minimal risks. Federal agencies have tried to address this problem by providing researchers and IRB members with some more guidance. The Food and Drug Administration (FDA) and the Department of Health and Human Services (DHHS) have published lists of procedures, tests, and examinations that they regard as routine. Some of these include: venipuncture; collecting of hair, placenta at delivery, sweat, saliva, or dental plaque; exercise by health volunteers; voice recordings; and recording of data using non-invasive procedures routinely employed in clinical practice, such as electrocardiography (ECG) and electroencephalography (EEG). ${ }^{27}{ }^{28}$ For this list to be effective, it must be updated frequently, because medicine and technology are constantly changing. For example, 20 years ago magnetic resonance imaging was not routine, but today it would probably be considered routine.

\section{CONCLUSION: REFORMING MINIMAL RISK}

The US regulations do not clearly define minimal risk. As a result of this ambiguity, different researchers and IRBs may 
interpret the concept differently. Although some commentators regard the ambiguity (or flexibility) of minimal risk as a virtue of the concept, others, myself included, regard it as a weakness. Lack of clarity concerning the interpretation of minimal risk can have an adverse impact on the consistency, fairness, and integrity of human research and can lead to the exploitation of vulnerable subjects.

What should be done to resolve the ambiguity? Kopelman has convincingly argued for an absolute interpretation of daily life risks to resolve the ambiguity in this part of the concept of minimal risk. However, this proposed solution does not eliminate the problem of identifying and assessing the daily life risks faced by a normal, healthy person. Although most people have a general sense of these risks, a general sense is not good enough. Researchers and IRB members need a clear and unambiguous definition of these risks as well. As long as the daily life risks standard remains a part of the definition of minimal risk, the definition of minimal risk will be ambiguous.

I propose that the easiest way clarify the concept of minimal risk would be to eliminate the daily life risks standard from the definition. Thus, minimal risk would be "the risks associated with routine physical or psychological exams". The latest version of the Council for International Organizations of Medical Sciences (CIOMS) guidelines adopts a similar definition. ${ }^{29}$ To provide additional guidance for researchers and IRB members, US federal agencies should provide IRBs with an extensive and diverse list of routine physical or psychological exams, and they should update this list periodically as new tests and exams become available.

The only disadvantage I can see from eliminating the daily life standard from the definition of minimal risk would be that this would deny IRBs some flexibility and adaptability that they need to review research. For example, it may very difficult to compare the aggregate risks of a novel or complex study with the risks associated with medical or psychological tests. To assess these novel or complex studies, one might argue, it is necessary to be able to appeal to a general concept of minimal risk, such as the daily life risks standard. Although I can see how the daily life risks standard may be useful in some situations, the problems associated with this part of the definition of minimal risk far outweigh any potential benefits. Promoting fairness, consistency, and justice in research is more important than promoting flexibility and adaptability.

\section{ACKNOWLEDGEMENTS}

The author would like to thank Loretta Kopelman, John Davis, and Ken De Ville for useful comments and suggestions.
Competing interest: I have no competing interests to declare.

The ideas and opinions expressed in this article are the author's personal views and do not represent the views of the National Institute of Environmental Health Sciences, the National Institute of Health, or the US government.

\section{REFERENCES}

145 CFR 46.102(i). See also 21 CFR 50.3(k).

2 Freedman B, Fuks A, Weijer C. In loco parentis: minimal risk as an ethical threshold for research upon children. Hastings Cent Rep 1993;23:13-19.

3 Kopelman L. Moral problems in assessing research risk. IRB 2000;22:3-7.

4 National Commission for the Protection of Human Subjects of Biomedical and Behavioral Research. 1979. The Belmont Report, Available at: http:// ohrp.osophs.dhhs.gov/humansubjects/guidance/belmont.htm (accessed 19 December 2003).

5 Levine R. Ethics and the regulation of clinical research. New Haven: Yale University Press, 1986.

6 Weijer C. Thinking clearly about research risk: implications of the work of Benjamin Freedman. IRB 1999;21:1-5.

7 Nelson R. Children as research subjects. In: Kahn J, Mastroianni A, Sugarman J, eds. Beyond consent: seeking justice in research. New York: Oxford University Press, 1998:47-67.

8 Kopelman L. Children as research subjects: a dilemma. J Med Philos 2000;25:745-64.

9 45.CFR 46.404

1045 CFR 46.405.

1145 CFR 46.406.

1245 CFR 46.407.

1345 CFR 46.404.

1445 CFR 46.306(a)

1545 CFR 46.207

1645 C.F.R. $46.116(d)$.

1745 CFR 46.117(c)(2)

1845 CFR 46.110(b); see also 21 CFR 56.110.

19 Janofsky J, Starfield. Assessment of risk in children. J Pediatr 1981;98:842-6.

20 Shah S, et al. How do institutional review boards apply the federal risk and benefit standards for pediatric research? JAMA 2004;291:476-82.

21 Kopelman L. Estimating risk in human research. Clin Res 1981;29:1-8.

22 Resnik D. Exploitation in biomedical research. Theor Med Bioeth 2003;24:233-59

23 Emanuel E, Wendler D, Grady C. What makes clinical research ethical? JAMA 2000;283:2701-11.

24 Grimes v Kennedy Krieger Institute, Inc, 782 A.2d 807 (Md. 2001). In the only other case to address minimal risk in research, a court ruled that a surrogate decision-maker may not consent to greater than minimal risk, non-beneficial research for a child or mentally disabled adult. See TD $v$ New York State Office of Mental Health, 650 NYS 2d 173 (1996).

25 National Bioethics Advisory Commission (NBAC). Ethical and Policy Issues in Research Involving Human Participants. Washington, DC: NBAC, 2001.

26 Weijer C. The ethical analysis of risk. J Law Med Ethics 2000;28:344-60.

27 Food and Drug Administration. Clinical investigations which may be reviewed through expedited review procedures set forth in FDA regulations. 46 Federal Register 8980 (27 January 1981).

28 Department of Health and Human Services. Categories of research that may be reviewed by the institutional review board (IRB) through an expedited review procedure. 63 Federal Register 216 (9 November 1998).

29 Council for International Organizations of Medical Sciences (CIOMS). International Ethical Guidelines for Biomedical Research Involving Human Subjects. 2002. Available at: http://www.cioms.ch/ frame_guidelines_nov_2002.htm (accessed 18 December 2003). 